\title{
Cryptococcus neoformans en la cavidad nasal de un gato en Costa Rica. Reporte de caso
}

\section{Cryptococcus neoformans in the nasal cavity of a cat in Costa Rica. A case report}

\author{
Andrea Urbina-Villalobos ${ }^{1 凶}$, Isabel Hagnauer², Noelia Méndez ${ }^{3}$, Natalia Guzmán-Gómez ${ }^{1}$, Alejandra \\ Calderón-Hernández ${ }^{1}$, Juan Alberto Morales ${ }^{4}$
}

1 Laboratorio de Micología, Escuela de Medicina Veterinaria, Universidad Nacional, Costa Rica. E-mail: andrea.urbina. villalobos@una.cr, natimichelle@hotmail.com, alejandra.calderon.hernandez@una.cr

2 Hospital de Especies Menores y Silvestres, Escuela de Medicina Veterinaria, Universidad Nacional, Costa Rica. E-mail: isabel.hagnauer.barrantes@una.cr

3 Clínica Veterinaria Dr. Gómez, Puntarenas, Costa Rica. noe.medvet.29@gmail.com

$4 \quad$ Servicio de Patología, Escuela de Medicina Veterinaria, Universidad Nacional, Costa Rica. juan.morales.acuna@una.cr

Recibido: 22 de mayo de 2018. Corregido: 14 de junio de 2018 Aceptado: 15 de junio de 2018.

Resumen: La criptococosis felina es causada por las levaduras encapsuladas de los complejos Cryptococcus neoformans y Cryptococcus gattii. Esta es la micosis sistémica más frecuente en felinos, los cuales pueden ser asintomáticos y presentar signos respiratorios o neurológicos. No han sido reportados casos en Costa Rica. Se recibió un paciente felino, macho, sin raza definida, pelo corto, de 4 años de edad, con historia de 6 meses de presentar estornudos y secreción nasal incolora, unilateral, refractaria a los tratamientos administrados. Se le realizó un lavado y una biopsia de mucosa nasal para análisis citológico y micológico. En la citología, se observó un proceso inflamatorio piogranulomatoso y gran cantidad de levaduras encapsuladas. Estas se observaron también en el examen microscópico directo con $\mathrm{KOH}$, tinta china y frotis teñido con Giemsa. En cultivo, se aisló Cryptococcus sp. y se confirmó C. neoformans, mediante API 20 C AUX (Biomérieux ${ }^{\circledast}$ ). Se administró tratamiento durante 9 meses y el gato evolucionó favorablemente. Se debe considerar la criptococosis en el diagnóstico diferencial de pacientes felinos con descargas nasales unilaterales incoloras y crónicas. La confirmación se debe realizar mediante pruebas de laboratorio para instaurar el tratamiento pertinente.

Palabras clave: criptococosis, Cryptococcus neoformans, gato, diagnóstico de laboratorio, micosis nasal.

Abstract: Feline cryptococosis is caused by the encapsulated yeasts of the complex Cryptococcus neoformans and Cryptococcus gattii. This is the most frequent mycosis in cats that could be either asymptomatic or with respiratory and neurologic signs. No cases have been reported in Costa Rica. A crossbreed, shorthair, male cat of 4 years old with refractory 6 months sneezing, unilateral nasal discharge, was examined. A biopsy of nasal mucosal tissue and a nasal lavage were performed in order to carry out cytological and mycological analysis. A pyogranulomatous process with several encapsulated yeasts were observed in the cytology. Encapsulated yeasts were also observed in the direct microscopic examination using $\mathrm{KOH}$, Indian Ink and in the smear stained with Giemsa. Cryptococcus sp. was isolated from tissue cultures and was confirmed as $C$. neoformans by API 20C AUX (Biomérieux ${ }^{\bullet}$ ). The cat was treated during 9 months with satisfactory clinical results. Cryptococcosis must be considered in the differential diagnosis of feline patients with chronic and colorless unilateral nasal discharge. Confirmation must be performed by laboratory tests in order to set up the appropriate treatment.

Keywords: cryptococcosis, Cryptococcus neoformans, cat, laboratory diagnosis, nasal mycosis.

$\square$

Autora para correspondencia: andrea.urbina.villalobos@una.cr 


\section{Introducción}

La criptococosis es una micosis sistémica causada por algunos hongos del género Cryptococcus. Existen diversas especies de esta levadura, aunque las especies más frecuentes que causan patología en felinos domésticos son de los complejos Cryptococcus neoformans y Cryptococcus gattii (Malik et al. 1997; Sykes \& Malik 2012). La enfermedad puede afectar a diferentes especies animales, pero se considera que el gato es la especie más susceptible, llega a ser reportada hasta 8 veces más que en perros (Duncan et al. 2006; Castellá et al. 2008; Stokes 2012; Sykes \& Malik 2012).

Cryptococcus neoformans es un hongo de distribución mundial y es el más común (Cabañes 2008), mientras que C. gattii es menos frecuente por estar limitado a zonas de clima tropical y subtropical; aunque actualmente se han reportado casos autóctonos en zonas de clima templado (Duncan et al. 2006; Springer \& Chaturvedi 2010).

El modo de infección de C. neoformans no está bien determinado; sin embargo, se considera que la principal ruta es mediante inhalación de basidiosporas o levaduras, directamente de ambientes contaminados con excretas de palomas y otras aves (Fooshee-Grace 2010; Stokes 2012). Rara vez ocurre infección localizada secundaria a heridas cutáneas penetrantes (Sykes \& Malik 2012). Tanto C. neoformans como C. gattii se encuentran en forma de levadura encapsulada en su fase parasitaria (Sykes \& Malik 2012).

Cryptococcus neoformans se considera un agente oportunista, pero en gatos rara vez se logra identificar una condición predisponente (Malik et al. 1997; Sykes \& Malik 2012). La sinología desarrollada depende de la localización de la infección. La cavidad nasal se considera el sitio primario de infección en felinos, por tanto, algunos signos frecuentes son rinitis, descarga nasal unilateral o bilateral, estornudos y linfonodos submandibulares aumentados de tamaño (Castellá et al. 2008; López 2012). En algunos casos, se pueden presentar estertores, distorsión facial y masas visibles en el interior de la nariz (Fooshee-Grace 2010; Johnson 2010). La infección puede llegar a vías aéreas inferiores, aunque esto es poco común (Johnson 2010), es posible que tenga presentación diseminada y afectando el ojo, piel, sistema nervioso central, entre otros órganos (Hawkins 2010; Sykes \& Malik 2012).

\section{Reporte de caso}

Un paciente felino macho, sin raza definida, pelo corto, de $4 \mathrm{~kg}$ de peso corporal y 4 años de edad, ingresó al Hospital de Especies Menores y Silvestres de la Universidad Nacional (HEMS-UNA) de Heredia, Costa Rica, referido porque había presentado un cuadro de estornudos y secreción nasal unilateral por 6 meses, sin respuesta positiva a corticoesteroides ni antibióticos y sin cambios relevantes en las radiografías faciales.

En el examen físico general, el paciente presentaba una buena condición corporal, los parámetros fisiológicos eran normales y se observó una secreción nasal incolora unilateral derecha. Con base en estos hallazgos y en la historia clínica, se establecieron 3 diagnósticos diferenciales: cuerpo extraño, micosis y neoplasia. Se realizó un lavado nasal para análisis citológico, en el Servicio de Patología, y una biopsia a ciegas de la mucosa nasal, de aproximadamente $2 \mathrm{~mm}$, para examen micológico en el Laboratorio de Micología de la UNA. 
En la citología del sedimento del lavado nasal teñido con Giemsa, se observó un proceso inflamatorio piogranulomatoso con presencia de neutrófilos, macrófagos y gran cantidad de levaduras encapsuladas, algunas en gemación. No se observaron células neoplásicas.

Para el análisis micológico, a la biopsia se le añadió solución salina con antibiótico (200 UI de penicilina y $200 \mu \mathrm{g} / \mathrm{ml}$ de estreptomicina), luego fue resuspendida y centrifugada (1500 rpm por 5 minutos). Se descartó el sobrenadante, el sedimento se resuspendió y se realizaron tres exámenes microscópicos directos, uno con hidróxido de potasio al $10 \%(\mathrm{KOH})$; otro con tinta china y el tercero con tinción Giemsa y se observaron en el microscopio de luz a 20X y 40X. En estos exámenes, se notaron abundantes levaduras encapsuladas con un halo incoloro (Fig. 1).

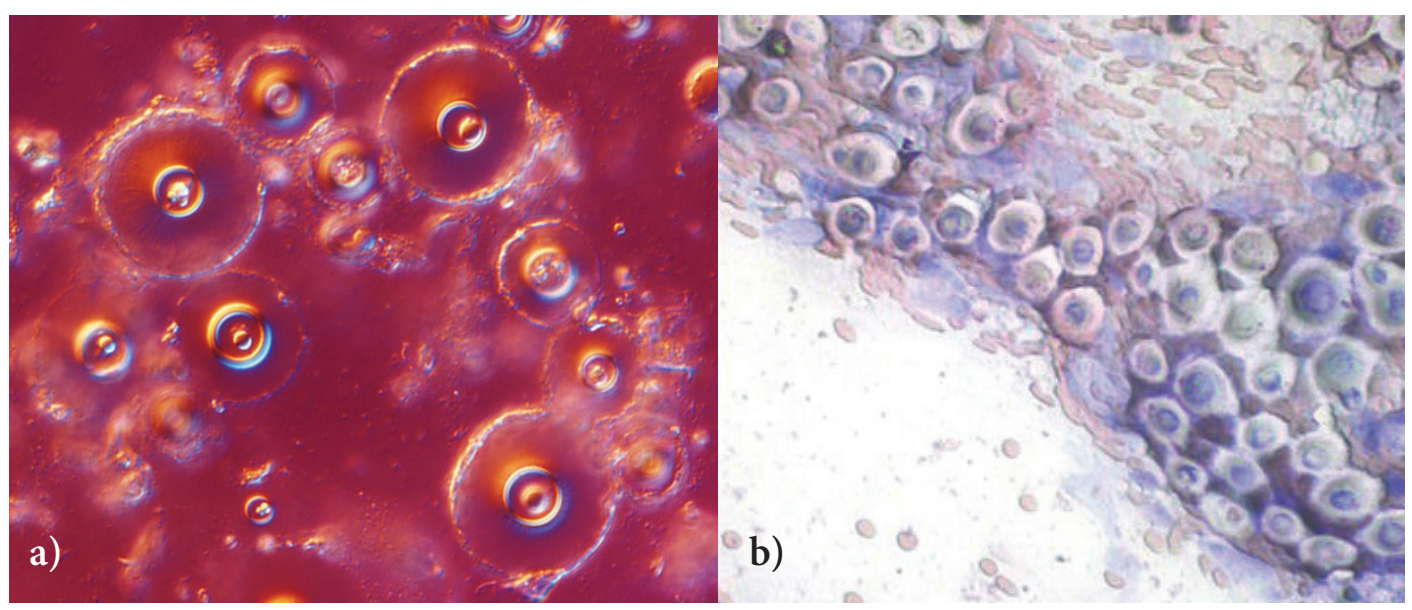

Figura 1. Examen microscópico directo de biopsia de mucosa nasal felina; a) KOH levaduras encapsuladas, Normarski Differential Interference Contrast 400x, b) tinción de Giemsa, abundantes levaduras encapsuladas $100 \mathrm{X}$

Otra porción del sedimento se cultivó en agar Sabouraud (Oxoid, Basingstoke, Reino Unido) a $37^{\circ} \mathrm{C}$ y $28^{\circ} \mathrm{C}$. En el cultivo, a las 72 horas de incubación a $37^{\circ} \mathrm{C}$, se aislaron incontables UFC levaduriformes de aspecto cremoso y mucoide. Las levaduras aisladas fueron observadas mediante tinción de Gram a 40X y 100X e identificadas utilizando el kit comercial API 20C AUX (BioMérieux, Marcy-l'Étoile, Francia), con el cual se identificó la levadura como Cryptococcus neoformans con una probabilidad de $99.5 \%$ (perfil bioquímico 2547173). Una vez establecido el diagnóstico, el gato fue tratado con Fluconazol, en una dosis de $10 \mathrm{mg} / \mathrm{kg}$ de peso, cada 12 horas, durante 9 meses. Al final del tratamiento, no se evidenció ningún signo asociado a criptococosis y se consideró clínicamente curado, ya que el propietario se negó a realizar otra biopsia de control.

\section{Discusión}

La criptococosis en animales, independientemente de su etiología, no representa una fuente de contagio ni para las personas ni para otros vertebrados (Cabañes 2008; Castellá et al. 2008). El diagnóstico 
oportuno en felinos tiene buen pronóstico y la precisión del médico en aquel, sumada al manejo terapéutico, tiene mucha importancia (Castellá et al. 2008), por lo que el clínico debe estar atento a los signos, para su confirmación.

Puede haber animales que tengan solo colonización nasal (Malik et al. 1997) o una infección asintomática, por tanto, una regla básica es realizar el diagnóstico definitivo basándose en la presencia de sinología clínica y pruebas de laboratorio que demuestren la existencia del agente etiológico en el tejido afectado (Malik et al. 1997; Castellá et al. 2008; Hawkins 2010). Además, hay poca información acerca de la epidemiología y casuística de la criptococosis en animales domésticos, por lo que felinos con signos de infección respiratoria superior con secreción o granulomas nasales deben ser estudiados por Cryptococcus spp. En el caso reportado, se logró la confirmación de la enfermedad con base en pruebas de laboratorio realizadas a la biopsia de mucosa y lavado nasales. La prueba de $\mathrm{API}^{\circ} 20 \mathrm{C}$ AUX indicó que era $C$. neoformans con alta probabilidad. Sin embargo, es importante hacer el diagnóstico diferencial con C. gattii, patógeno primario emergente con distribución geográfica creciente (Springer \& Chaturvedi 2010). Para diferenciar C. neoformans de C. gattii lo más recomendable es usar el medio L- canavanina- glicina-azul de bromotimol en conjunto con secuenciación del ADN (Klein et al. 2009).

\section{Conclusión}

Se debe considerar criptococosis en el diagnóstico diferencial de pacientes felinos con descargas nasales incoloras y crónicas. La confirmación se debe realizar mediante pruebas de laboratorio para instaurar el tratamiento pertinente.

\section{Conflicto de intereses}

Los autores declaran no tener conflicto de intereses.

\section{Referencias}

Cabañes, F. J. 2008. Micosis y zoonosis: Cryptococcus spp. Rev. Iberoam. Micol. 25: S1-3. Doi:10.1016/ S1130-1406(08)70018-4

Castellá, G., Abarca, M. L. \& Cabañes, J. 2008. Criptococosis y animales de compañía. Rev. Iberoam. Micol. 25: S19-24. Doi: 10.1016/S1130-1406(08)70021-4

Duncan, C., Stephen, C. \& Campbell, J. 2006. Clinical characteristics and predictors of mortality for Cryptococcus gattii infection in dogs and cats of Southwestern British Columbia. Canadian Vet. J. 47(10): 993-98.

Fooshee-Grace, S. 2010. Cryptococcosis. In: Norsworthy, G. D., Fooshee-Grace, S., Crystal, M. A. \& Tilley, L. P. (Ed.). The feline patient, $4^{\text {th }}$ Edition. Wiley-Blackwell, Iowa. p. 97-99.

Hawkins, E. C. 2010. Respiratory system disorders: clinical manifestation of nasal disease. In: Nelson, R. W. \& Couto, C. G. (Ed.). Small animal internal medicine. Elsevier, Missouri. p. 217-23.

Johnson, L. R. 2010. Nasal disorders. In: Clinical canine and feline respiratory medicine. Wiley- 
Blackwell, Iowa. p. 65-68.

Klein, K. R., Hall, L., Deml, S. M., Rysavy, J. M., Wohlfiel, S. L. \& Wengenack, N. L. 2009. Identification of Cryptococcus gattii by use of L-canavanine glycine bromothymol blue medium and DNA sequencing. J. Clin. Microbiol. 47(11): 3669-72. Doi: 10.1128/JCM.01072-09

López, A. 2012. Respiratory system, mediastinum and pleurae. In: Zachary, J. \& MacGavin, M. D. (Ed.). Pathologic basis of veterinary disease, $5^{\text {th }}$ Edition. Elsevier-Mosby, Missouri. p. 529-30.

Malik, R., Wigney, D. I., Muir, D. B. \& Love, D. N. 1997. Asymptomatic carriage of Cryptococcus neoformans in the nasal cavity of dogs and cats. J. Med. Vet. Mycol. 35: 27-31.

Springer, D. J. \& Chaturvedi, V. 2010. Projecting global occurrence of Cryptococcus gattii. Emerg. Infect. Dis. 16(1): 14-20. Doi: 10.3201/eid1601.090369

Stokes, J. 2012. Infectious diseases. Fungal and rickettsial diseases. In: Little, S. (Ed.). The Cat: Clinical Medicine and Management. Elsevier Saunders, Missouri. p.1016 -19.

Sykes, J. E. \& Malik, R. 2012. Cryptococcosis. In: Greene, C. E. (Ed.). Infectious diseases of the dog and cat, $4^{\text {th }}$ Edition. Elsevier-Saunders, Missouri. p. 621-34. 